\title{
Introduction to "Analyzing the Impact of Digitization on Business Operations" Minitrack
}

\author{
Abraham Seidmann \\ Primary Minitrack Chair \\ W. E. Simon Graduate School of \\ Business Administration \\ University of Rochester \\ avi.seidmann@simon.rochester.edu
}

\author{
Yabing Jiang \\ Minitrack Chair \\ Lutgert College of Business \\ Florida Gulf Coast University \\ yjiang@,fgcu.edu
}

\author{
Jie Zhang \\ Minitrack Chair \\ College of Business Administration \\ University of Texas at Arlington \\ jiezhang@uta.edu
}

This year "Analyzing the Impact of Digitization on Business Operations" minitrack at HICSS has accepted six papers, which explore the increasing complex roles that advanced information technologies play in healthcare, education, business operations, and consumer decisions and activities. The accepted papers are as follows:

- Digitization in B2B Buying and Selling: This paper empirically examines the ways in which transacting buyers and suppliers use different digital technologies for each of these processes. Given the bilateral nature of transactions, it also examines for which processes buyers and suppliers are misaligned in their use of digital technologies. This field study is based on surveys of transacting agents in 174 buyersupplier dyads about their use of digital technologies. The results indicate a number of significant differences in digital process support by buyers and suppliers that firms can address for improved transaction efficiency.

- Identification of Institutional Characteristics that May Drive (Avoidable) Hospitalization of Nursing Home Residents: Avoidable hospital transfers increase the risk of negative outcomes for patients and cause waste in medical resources. The paper, rooted in the context of nursing homes, provides a comprehensive review of the concept, identification, and contributing factors. In addition, empirical work using nationwide aggregate data is presented to complement the study. The authors found that while caregiver/resident ratio remains a heated topic in public and policy debate, only a high intensity of service provided by well-trained medical practitioners (registered nurses and physicians) is associated with low levels of avoidable ED visits and hospitalization.

- $\quad$ The Impact of Digital Technology on Network Value Co-creation: The interplay of the introduction of new technologies and the network value cocreation (VCC) for small and medium-sized enterprises (SME) has not been well explored. To fill this research gap, the authors conducted a single longitudinal embedded case study in a network of four German specialist textile companies undergoing digital transformation. They identified three key technologies: a service configuration system, a centralized knowledge base, and an analytics system for promoting network VCC. The case study examined how company networks introduce new technologies for enhanced VCC and how these technologies impact the network VCC. It offered practical examples of how the transformation of $\mathrm{VCC}$ in SME networks can be realized.

- When Fake Reviews are Funny, Who Has the Last Laugh?: Consumers are increasingly relying upon online reviews to guide their decision-making. This paper empirically examines the impact of a specific type of fake reviews, the funny reviews, on consumer engagement and sales. The authors found that while funny reviews increase consumer engagement, they also decrease purchase intention compared to positive non-funny reviews. This study extends the online reviews literature and offers practical implications for sellers and platform providers dealing with all sorts of fake reviews.

- $\quad$ Minimizing Response Time of IoT-Based Traffic Information System through Decentralized Server System: Many metropolises seek to relieve traffic congestions and reduce vehicle accidents by implementing Intelligent Traffic Information Systems. These systems manage continuous communication between vehicles, various roadside IoT devices and a central server in real time for traffic control and vehicle navigations. Short response time is critical to the success of these timesensitive systems. For a small area, a system with centralized server architecture may just work fine. For a larger area with more IoT devices and traffic, however, the system may experience excessive response time as a result of increased network 
distance and constrained server processing capacity. The authors propose a decentralized server system to properly manage and reduce service response time. They have also developed a binary nonlinear constrained programming model with Genetic Algorithm for a heuristic solution.

- Predicting Students' College Drop Out and Departure Decisions by Analyzing their CampusBased Social Network Text Messages: Higher education plays a critical role training and preparing future workforce in every field. And yet, nearly $50 \%$ of students who enrolled in colleges will not graduate. This paper aims to tackle the undergraduate retention issue through big data analysis. The authors developed a text mining model, analyzing student app text messages. Through identifying the differences and trends of language used by students who were retained for the first year and those who dropped out, the proposed model will help predict undergraduate student dropouts and facilitate schools allocating retention resources and developing a more effective drop out intervention program. 